\title{
Reconstruction of NSTX midplane neutral density profiles from visible imaging data
}

\author{
D. P. Stotler ${ }^{a, *}$, F. Scotti ${ }^{b}$, R. E. Bell ${ }^{a}$, B. P. LeBlanc ${ }^{a}$, R. Raman ${ }^{c}$ \\ ${ }^{a}$ Princeton Plasma Physics Laboratory, Princeton University, P. O. Box 451, \\ Princeton, NJ 08543-0451, USA \\ ${ }^{b}$ Lawrence Livermore National Laboratory, Livermore, CA 94551, USA \\ ${ }^{c}$ University of Washington, Seattle, WA 98195, USA
}

The experimental determination of neutral densities in tokamak plasmas from line radiation is only accurate in the narrow region in which both the excitation rate and neutral density are significant. We describe an alternative procedure using the DEGAS 2 Monte Carlo neutral transport code to invert light emission data obtained from a tangentially viewing camera, yielding absolute radial profiles of deuterium atoms and molecules at midplane. That the neutral source in these simulations can be adequately characterized as a uniform flux at the vacuum vessel wall is demonstrated by the similarity of the shapes of the simulated and observed brightness profiles. A second test is obtained by comparing the resulting neutral pressures at the vessel walls with data from midplane micro-ion gauges. We also show that the simulated camera image is insensitive to variations in the spatial distribution of the neutral source.

Keywords: (PSI-21) NSTX DEGAS Neutrals Edge Modeling Spectroscopy PACS 52.25.Ya 52.55.Fa 52.70-m 52.65.Pp

${ }^{*}$ Corresponding and presenting author

Email address: dstotler@pppl.gov (D. P. Stotler) 


\section{Introduction}

The densities of neutral atoms and molecules in the main chamber of tokamaks are required to estimate their effects on particle, momentum, and en-

ergy balance (e.g., [1]), on the formation of the H-mode pedestal [2], and on the stabilization of plasma turbulence [3]. Midplane neutral densities in particular are needed to determine charge exchange losses of neutral beam ions $[4,5]$; to quantify fluxes of energetic charge exchange atoms to the main chamber wall, along with the associated sputtering [6]; and in the interpretation of edge diagnostics [e.g., charge exchange recombination spectroscopy (CHERS) [7]].

Multiple measurements of the neutral deuterium atom density have been made previously $[1,8,9,10]$. The most common technique infers the density via an inversion of the light measured by a calibrated camera. For example, Ross [5] used the Balmer- $\beta$ line with the volumetric rate of light emission $S_{\beta}$ being given by:

$$
S_{\beta}=n_{\mathrm{D}}(1 s)\left[\frac{n_{\mathrm{D}}(n=4)}{n_{\mathrm{D}}(1 s)}\right] A_{4 \rightarrow 2} \equiv n_{\mathrm{D}} F\left(n_{e}, T_{e}\right),
$$

where $n_{\mathrm{D}}(1 s)$ is the density of the deuterium ground state, the function $F$, representing the ratio of the density of the upper state of the transition to the ground state, is obtained from a collisonal radiative model [11], and $A_{4 \rightarrow 2}$ is the Einstein coefficient for the transition. If $n_{e}$ and $T_{e}$ are available from some other diagnostic covering the same volume in which $S_{\beta}$ is measured, the value of $F$ can be determined, giving

$$
n_{\mathrm{D}}=S_{\beta} / F\left(n_{e}, T_{e}\right)
$$


The first difficulty with this approach is that $S_{\beta}$ and $F$ are significant only over a relatively narrow radial region of the edge plasma. Farther out, where $n_{\mathrm{D}}$ is largest, $n_{e}$ and $T_{e}$ are too low to excite the upper state of the transition; farther in, $n_{\mathrm{D}}$ is reduced by ionization. In both cases, the signal $S_{\beta}$ is small and likely dominated by noise. The second difficulty is in determining $S_{\beta}$ from the line integrated measurements. Doing so via Abel inversion [12] requires assuming that $S_{\beta}$ is only a function of major radius.

Direct modeling of the main chamber neutral density is also problematic since the dominant source of neutral molecules is usually recycling at the divertor targets. The first step in reconstructing that source is assembling a consistent characterization of the divertor plasma constrained by the available diagnostic data, a time consuming task that may or may not yield a satisfactory result [13]. Once this is done, one would need to simulate the flow of the neutrals through the scrape-off layer (SOL) plasma to the vicinity of the midplane. Because the far SOL plasma is poorly diagnosed in most tokamaks, only rough estimates can be made of the attenuation of the neutral flux due to ionization. Furthermore, the main chambers of most tokamaks are geometrically complex, possessing 3-D vessel structures behind which neutrals can flow (Fig. 1). Main chamber recycling of large amplitude "blobs" striking the vessel wall represents another potentially significant source of neutrals [14]. Because this source is inadequately characterized and possibly toroidally asymmetric, estimates of its magnitude are highly uncertain.

We describe here a hybrid approach in which a DEGAS 2 [15] neutral transport simulation is used to augment the experimental procedure, extending the spatial range over which useful densities can be obtained. Similar 
methods were employed in $[1,8,10]$. The use of 2-D or 3-D neutral transport simulations eliminates the need for Abel inversion, allowing us to account for toroidal asymmetries and the detailed path of the camera views through the plasma. We will demonstrate the improvement over the use of Eq. (2) in Sec. 3. Our confidence in the accuracy of the method stems from the successful application of a similar technique to the quantitative simulation of the neutral gas cloud in NSTX midplane gas puff imaging experiments [16, 17].

Section 2 describes the simulation technique and the underlying assumptions. Its application to two NSTX discharges is presented in Sec. 3, including the tests which constitute an initial confirmation of the method. The sensitivity of the profiles to the spatial variation of the neutral source, a principal assumption of the method, is examined in Sec. 4. Finally, in Sec. 5, we summarize and present topics for further investigation.

\section{Simulation Method}

The primary diagnostic employed in the present analysis was originally designed to allow a simple inversion via Eq. (2) [5]. The Edge Neutral Density Diagnostic (ENDD) uses an absolutely calibrated camera with a tangential view through the edge of the NSTX plasma, providing radial profiles with $1.6 \mathrm{~mm}$ spatial resolution. The exposure time for each frame is $3.7 \mathrm{~ms}$, or 268 frames per second. The camera image has $128 \times 127$ pixels, although vignetting and light reflections restrict this view to 123 pixels in the radial direction $(20 \mathrm{~cm})$, and 66 pixels poloidally $(9 \mathrm{~cm})$. As will be discussed in Sec. 4 , variation of the signal in this poloidal direction is small and generally 
ignored. The location of the ENDD view relative to other in-vessel structures is indicated schematically in Fig. 1.

As in the GPI simulations [16, 17], the DEGAS 2 geometry is based on contours of constant poloidal magnetic flux, from an NSTX EFIT $[18,19]$ equilibrium, drawn inside a toroidally axisymmetric rectangle encompassing the emission volume viewed by the ENDD camera. The outer boundary of this rectangle corresponds to the vacuum vessel wall (Fig. 1); the inner boundary at $R=1.2 \mathrm{~m}$ is inside the penetration depth of all but the most energetic atoms. The passive plates are too far from the ENDD field of view for recycling there to contribute to its signal. We thus exclude them from the simulation box by limiting its vertical extent to $Z=-0.40 \rightarrow 0.54 \mathrm{~m}$, simplifying the geometry in the process.

The electron density and temperature profiles input to DEGAS 2 are derived from Thomson scattering data as in [16], except that we now use the CHERS diagnostic to estimate values for $n_{D}^{+} / n_{e}$ and $T_{i} / T_{e}$. For the shots described in this paper, $T_{i}$ does not differ from $T_{e}$ significantly, and we assume $T_{i}=T_{e}$. The plasma profiles are assumed to be radially constant outside the outermost Thomson scattering point $(R=1.56 \mathrm{~m})$.

The results in Sec. 3 are from 3-D simulations extending 103 degrees toroidally, 15 degrees beyond the ENDD view in both directions. This geometry incorporates the Scintillator Fast Lost Ion Probe (sFLIP) [20] diagnostic just below the ENDD field of view (Fig. 1), but not the neutral beam armor. Since the sFLIP plate may serve as an additional, nearby, source of recycled neutrals, we have incorporated it to estimate its effect. The beam armor is further away and is expected to make less of a contribution. The sensitivity 
calculations discussed in Sec. 4 are axisymmetric and do not contain either structure. Some conclusions regarding the dimensionality of the problem will be drawn in Sec. 5.

The sFLIP structure in the 3-D runs is also used to shield a portion of the simulation volume from the plasma to allow for the determination of a vacuum vessel density that can be directly compared with data from microion gauges that are similarly shielded [21]. To get corresponding values in axisymmetric runs, a $2 \mathrm{~cm}$ wide slot is introduced $3 \mathrm{~cm}$ below midplane [Fig. 1(b)].

The primary difference between the present technique and that described in $[16,17]$ is that the neutral source is not known here. In the absence of any additional information, we simply postulate a uniform, axisymmetric source of deuterium molecules coming off of the vacuum vessel walls with a thermal distribution at $300 \mathrm{~K}$ and a cosine angular distribution. The flux from this surface is assigned an arbitrary magnitude of $10^{20} \mathrm{D}_{2} /\left(\mathrm{s} \mathrm{m}^{2}\right)$. In the 3-D simulations, the same is done for the surface of the sFLIP plate. We will demonstrate in Sec. 4 that the simulated ENDD signal is insensitive to the spatial distribution of the source.

The atomic physics model used in these simulations is the same as that in [16] with two modifications. First, the volumetric source of $\mathrm{D}_{\beta}$ photons, computed via Eq. (1), is accumulated in each computational zone along the atoms' paths; a synthetic ENDD image is constructed from these data in post-processing. Second, we have updated the collisional radiative model used in [16], which was based on [22] with cross sections from [23], to incorporate new $n=1 \rightarrow 3,4$, and 5 excitation cross sections obtained from 
Convergent Close Coupling calculations [24]. The $\mathrm{D}_{\beta}$ emissions from molecular and molecular ion dissociation are presently ignored.

The results described in Sec. 3 are obtained by comparing a single horizontal row of pixels at the center of the simulated ENDD signal, $9 \mathrm{~cm}$ above midplane, with a corresponding row from the experimental imaged smoothed by binning over 10 adjacent vertical pixels $(1.4 \mathrm{~cm})$ to mitigate electronic noise from the camera. The ratio of the peak brightness in the ENDD signal, in photons / $\left(\mathrm{s} \mathrm{sr} \mathrm{m}^{2}\right)$, to that of the DEGAS 2 simulated signal provides an overall scale factor that can then be applied to all of the DEGAS 2 output, via the linearity of the neutral transport system, and to the neutral atom and molecular densities, in particular.

The integration time of the ENDD camera of $3.7 \mathrm{~ms}$ is long compared to blob transport time scales and can even encompass multiple small scale ELMs (NSTX type V ELMs have frequencies in the $300-800 \mathrm{~Hz}$ range [25]). Hence, the ENDD signal incorporates the effects of both when present. In this work, however, we make no attempt to account for them; all calculations assume steady state.

\section{Results}

We apply this method to the H-mode phases of two 2010 NSTX shots, 139412 and 142214, both at $0.4 \mathrm{~s}$ into the discharge. Shot 139412 had relatively low triangularity (0.3) and exhibited considerable ELM activity; the selected time is during a lull in that activity $\left(B_{T}=0.47 \mathrm{~T}, I_{p}=0.79 \mathrm{MA}, q_{95}=9.7\right.$, maximum $\left.n_{e}=4.7 \times 10^{19} \mathrm{~m}^{-3}, T_{e}=980 \mathrm{eV}\right)$. Shot 142214 had medium 
triangularity (0.6) and was ELM free $\left(B_{T}=0.54 \mathrm{~T}, I_{p}=0.82 \mathrm{MA}, q_{95}=\right.$ 8.1, maximum $\left.n_{e}=4.6 \times 10^{19} \mathrm{~m}^{-3}, T_{e}=1400 \mathrm{eV}\right)$. Neither of these shots was preceded by fresh lithium evaporation [26]. However, the campaignintegrated in-vessel evaporation for 142214 was much larger, $800 \mathrm{~g}$, than that for 139412, $300 \mathrm{~g}$. Both plasmas also had relatively high SOL densities for NSTX H-modes, $\sim 10^{18} \mathrm{~m}^{-3}$, allowing the Thomson scattering diagnostic to provide accurate $n_{e}$ and $T_{e}$ at all points. A future publication will consider a greater variety of discharges.

Figure 2 compares the resulting simulated ENDD profiles to those observed. Their similarity provides an initial confirmation of our approach to inverting the ENDD data and of the adequacy of the uniform $\mathrm{D}_{2}$ source ansatz. A more careful analysis of the simulation uncertainties that allows this comparison to be quantified will be presented elsewhere.

The overall scaling factor for the neutral source is found to be 2.5 (1.6) for 139412 (142214). The resulting scaled radial profiles of the $\mathrm{D}$ and $\mathrm{D}_{2}$ densities at midplane, extracted from the simulation volume, are shown in Fig. 3. The neutral density profile variation between the outermost Thomson scattering point $(R=1.56 \mathrm{~m})$ and the wall at $R=1.7 \mathrm{~m}$ depends on the model used to extrapolate the plasma parameters over this region (assumed constant); in principle, one could fit a parameterized profile (e.g., via scale lengths) to better match the outer portion of the ENDD profile.

We have included in Fig. 3 the D density profile for shot 142214 computed from Eq. (2), following an Abel inversion [12] to obtain $S_{\beta}$. The principal improvement of the DEGAS 2 method over Eq. (2) is the greater spatial range, at both smaller and larger major radii, over which density profile 
information is obtained. Secondly, the use of Eq. (2) provides no measure of the $\mathrm{D}_{2}$ density. Thirdly, the simulated profile is the result of a "global" (within the simulation volume) transport calculation so that the value at each radius is related to the others in a consistent manner. In contrast, the values of Eq. (2) are connected only via the Abel inversion procedure and may be significantly impacted by fluctuations in the ENDD signal. The $S_{\beta}(R)$ assumption required by the inversion technique is relatively well satisfied here and does not introduce significant errors.

We compare in Table 1 the simulated neutral densities at the vessel wall with those measured by neutral pressure gauges. One of these ("C-mid") is a micro-ion gauge [21] relatively close to the vessel, providing a fast measurement of the neutral density. On the other hand, the values of interest are near the lower end of the gauge's operating range, resulting in a significant uncertainty and order unity variations in pressure from one data point to the next. The second ("IG 110") is less noisy, but is farther away from the vessel. To account for the corresponding delay in its response, we shift its signal by $0.18 \mathrm{~s}$, determined by comparing the higher pressure, post-discharge signals of the gauges. To assess the implications of these limitations on our ability to obtain data for a particular shot, we have conducted a survey of the density readings for the two gauges (averaged over 0.1 seconds) over 17 shots, including 139412 and 142214. No clear correlation between the C-mid and IG 110 signals is found in this survey, implying that the data in Table 1 provide only an order of magnitude estimate of the density; an unambiguous trend between these two shots cannot be established. We believe that this is primarily a result of the low pressures involved and the distance between the 
plasma and the IG 110 gauge. The survey shows that the density readings for the two gauges vary over a limited range in the 17 shots, between $2 \times 10^{17}$ and $1 \times 10^{18}$.

As noted in Sec. 2, the DEGAS 2 density values in the baseline 3-D simulations are taken in a slot behind the sFLIP plate, and we are not attempting to replicate the conductance paths between the simulation volume and the location of the actual pressure gauges. To estimate the attendant uncertainty in the vessel densities, we have included in Table 1 values from axisymmetric runs with an alternative slot configuration [Fig. 1(b)]. The sFLIP slot densities are higher since source molecules on adjacent wall surfaces can find their way in without penetrating the plasma. In contrast, the density in the axisymmetric slot results almost entirely from atoms heading out from the plasma. Note that all four simulated values are in the $2 \times 10^{17}$ and $1 \times 10^{18}$ range obtained from the broader survey of pressure gauge data.

That the ratios of the 139412 densities to those for 142214 are similar to the ratio of the overall scaling factors quoted above $(\sim 1.6)$ is interesting. In fact, the "unscaled" vessel density values for each slot configuration are comparable for the two shots. We will examine this insensitivity in more detail in a subsequent publication.

\section{Sensitivity to Source Variations}

To assess the sensitivity of this method to the assumption of a uniform source along the vacuum vessel wall, we consider a $2-\mathrm{D}$ simulation in which the source flux drops from $2 \times 10^{20} \mathrm{D}_{2} /\left(\mathrm{m}^{2} \mathrm{~s}\right)$ at $Z=-0.4$ to $2 \times 10^{19} \mathrm{D}_{2} /\left(\mathrm{m}^{2} \mathrm{~s}\right)$ at 


\begin{tabular}{ccccc}
\hline Shot & C-mid & IG 110 & sFLIP slot & 2-D slot \\
\hline 139412 & 5 & 8 & 10.2 & 6.9 \\
142214 & 7 & 5 & 5.8 & 4.1 \\
\hline
\end{tabular}

Table 1: Molecular deuterium density (in units of $10^{17} \mathrm{~m}^{-3}$ ) from NSTX shots 139412 and 142214 as measured by two ionization gauges, one close to the plasma (C-mid) and one further away (IG 110), and as estimated from DEGAS 2-ENDD simulations, behind the sFLIP plate (3-D cases) or the adjacent axisymmetric slot (2-D).

$Z=0.54$, i.e., a factor of 10 variation over the vertical range of the problem. Such a source is intended to mock up a neutral "bath" flowing up from the lower divertor, but being attenuated en route by the far SOL plasma. The maximum relative deviation of the resulting normalized, simulated ENDD profile from the baseline (uniform source, 2-D) run is 3\%. The scaled D density profiles differ on average by only $12 \%$. For $\mathrm{D}_{2}$ the average deviation is $14 \%$, if we ignore larger deviations in the tail where the densities are $<10^{-5}$ of their maximum. We expect that such deviations are smaller than the overall uncertainties in the density profiles.

This spatially varying source does result in a vertical variation in the simulated 2-D ENDD image, about $10 \%$ in the vicinity of the peak and $20-30 \%$ in the far SOL. An examination of the ENDD images from shots 139412 and 142214 does suggest a similar up-down variation in the far SOL, but not near the peak. A more careful uncertainty analysis, including other shots, would be needed to determine the significance of this observation. The 3-D simulation of 142214 documented in Sec. 3 does exhibit vertical 
variation comparable to that in the experimental image just due to source on the sFLIP plate. Because the sFLIP source is closer to the plasma and angled towards the observation volume, it effectively acts as a source that is stronger in the lower half of the problem volume. However, the image from the 3-D simulation of 139412 is closer to up-down symmetric, suggesting that this effect is sensitive to the details of the poorly known far SOL plasma parameters.

More extreme spatial variations have been considered in which the source is placed at a particular location on the outer wall or lower boundary of an axisymmetric simulation. In all cases, the simulated ENDD profile closely resembles that of the baseline run shown in Fig. 2, with differences $<10 \%$ of the peak value. These deviations are smaller than those between the observed and simulated profiles, suggesting that the latter are not associated with the spatial variation of the source. We suspect that they are instead the result of our inability to accurately reconstruct the plasma profiles, principally due to the limited spatial and temporal resolution of the NSTX Thomson scattering system.

We can compare the axisymmetric and 3-D simulations to estimate the magnitude of toroidally distant sources. As was noted in Sec. 2, the source in the 3-D runs extends 103 degrees in the toroidal direction, compared with 360 for the axisymmetric simulations. The integrated source current in the axisymmetric case is, thus, a factor of 3.5 times greater. Nonetheless, the peaks in the simulated ENDD profiles differ by only $1.5 \%$. This suggests that the contributions from recycling off of the neutral beam armor are likely small and not worth the effort required to incorporate that structure into the 
simulation geometry.

\section{Conclusions}

We have described a method for using DEGAS 2 to infer full neutral density profiles near midplane using data from the NSTX ENDD diagnostic. The similarity of the simulated and observed ENDD profiles provides one check of the DEGAS 2-ENDD method. The agreement, albeit with significant uncertainties, of the simulated and observed vacuum vessel pressures serves as a second test. The simulated midplane neutral density profiles have been shown to be insensitive to the principal assumption in the technique, the spatial variation of the neutral source. The ENDD signal, thus, tells us more about the integrated source strength and the plasma profiles than it does about the source distribution. This result, combined with the insignificant contributions of the sFLIP source to the ENDD signal, suggest that the neutral penetration is effectively one-dimensional. However, a 2-D calculation is needed to accurately replicate the views of the ENDD camera and to estimate vacuum vessel pressures for comparison with micro-ion gauge data. On a modest size cluster (32 to 64 cores), such simulations can be run in a few minutes.

An independent test of the DEGAS 2-ENDD technique has been provided courtesy of an OEDGE reconstruction of NSTX shot 139396 [13]. The resulting midplane neutral density profiles matched those obtained via DEGAS 2-ENDD to within a factor of two.

A future publication will examine a wider range of discharges and quantify 
the uncertainties in the resulting profiles. The largest uncertainties are likely those associated with the specification of the plasma profiles, e.g., due to plasma turbulence, ELMs, or just extrapolation. The observed sensitivity of the simulated ENDD profiles to the assumed plasma profiles may provide a basis for inferring effective plasma profiles for these cases, allowing for a more realistic reconstruction of the neutral density profiles.

\section{Acknowledgments}

The authors wish to acknowledge P. W. Ross and A. L. Roquemore for the design, construction, and installation of the ENDD diagnostic. This work is supported by U.S. DOE Contracts DE-AC02-09CH11466 (PPPL), DEAC52-07NA27344 (LLNL), and DE-SC0006757 (University of Washington).

\section{References}

[1] R. L. Boivin et al., Phys. Plasmas 7 (2000) 1919.

[2] M. A. Mahdavi et al., Phys. Plasmas 10 (2003) 3984.

[3] D. A. Russell et al., Phys. Plasmas 16 (2009) 122304.

[4] S. S. Medley et al., Nucl. Fusion 44 (2004) 1158.

[5] P. W. Ross, Ion power balance in neutral beam heated discharges on the national spherical torus experiment (NSTX), Ph.D. thesis, Princeton University (2010), http://search.proquest.com/docview/520394282. 
[6] P. C. Stangeby, J. Nucl. Mater. 415 (2011) S278.

[7] R. E. Bell et al., Phys. Plasmas 17 (2010) 082507.

[8] R. D. Bengtson et al., Rev. Sci. Instrum. 61 (1990) 3110.

[9] R. J. Colchin et al., Nucl. Fusion 40 (2000) 175.

[10] J. Harhausen et al., Plasma Phys. Control. Fusion 53 (2011) 025002.

[11] L. C. Johnson and E. Hinnov, J. Quant. Spectrosc. Radiat. Transfer 13 (1973) 333.

[12] R. E. Bell, Rev. Sci. Instrum. 66 (1995), 558.

[13] J. H. Nichols et al., "OEDGE modeling of outer wall erosion in NSTX and the effects of changes in neutral pressure" (these proceedings).

[14] M. V. Umansky et al., Phys. Plasmas 5 (1998) 3373.

[15] D. P. Stotler, C. F. F. Karney, Contrib. Plasma Phys. 34 (1994) 392.

[16] B. Cao et al., Fusion Sci. Tech. 64 (2013) 29.

[17] D. P. Stotler et al., J. Nucl. Mater. 363-365 (2007) 688.

[18] L. L. Lao et al., Nucl. Fusion 25 (1985) 1611.

[19] S. A. Sabbagh et al., Nucl. Fusion 41 (2001) 1601.

[20] D. S. Darrow, Rev. Sci. Instrum. 79 (2008) 023502.

[21] R. Raman et al., Rev. Sci. Instrum. 74 (2003) 1900. 
[22] J. C. Weisheit, J. Phys. B 8 (1975) 2556.

[23] R. K. Janev, J. J. Smith, At. Plasma-Mater. Interaction Data Fus. 4 (1993) 1.

[24] I. Bray et al., Phys. Reports 520 (2012) 135.

[25] R. Maingi et al., Nucl. Fusion 45 (2005) 264.

[26] H. W. Kugel et al., Fusion Eng. Des. 84 (2009) 1125. 

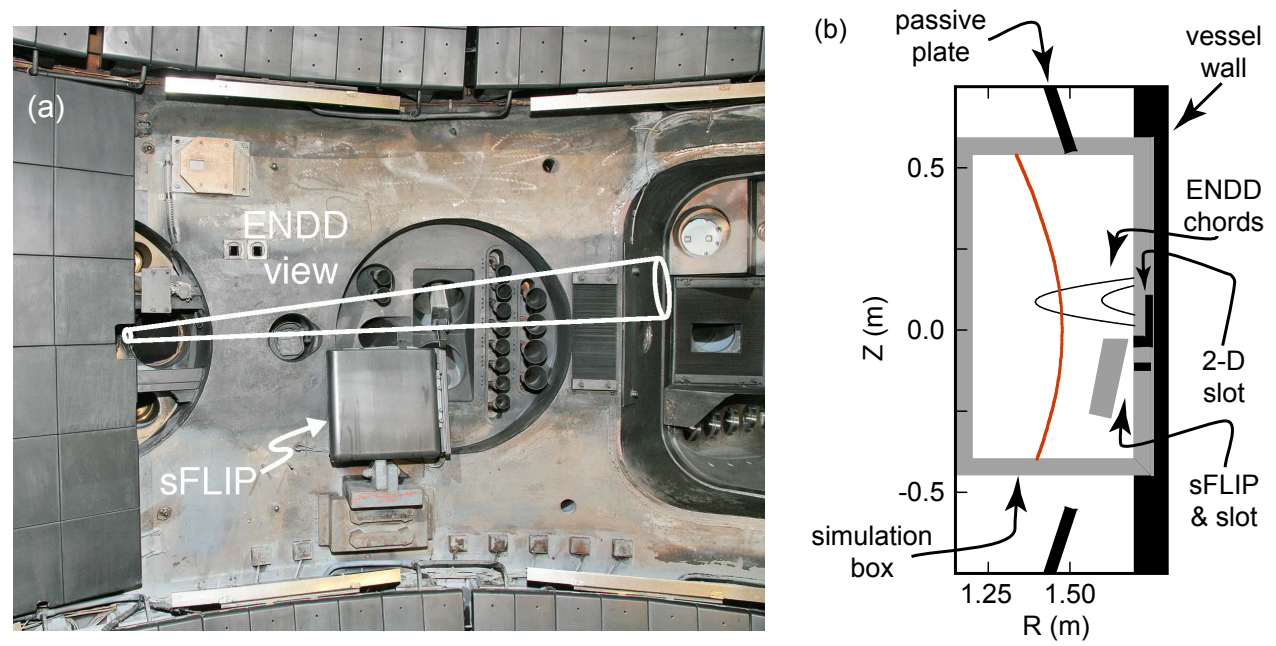

Figure 1: (a) Schematic representation of the view of the ENDD camera showing adjacent hardware structures, including the sFLIP diagnostic, passive plates (tiled surfaces upper and lower), and the neutral beam armor (on left). (b) Corresponding view in the poloidal plane, showing the relative locations of the separatrix (red; shot 139412), two ENDD chords (innermost and outermost from the central horizontal row), the sFLIP diagnostic with gas pressure slot, and gas pressure slot for axisymmetric simulations. The locations of the upper and lower passive plate tiles (not simulated) are included for comparison. 

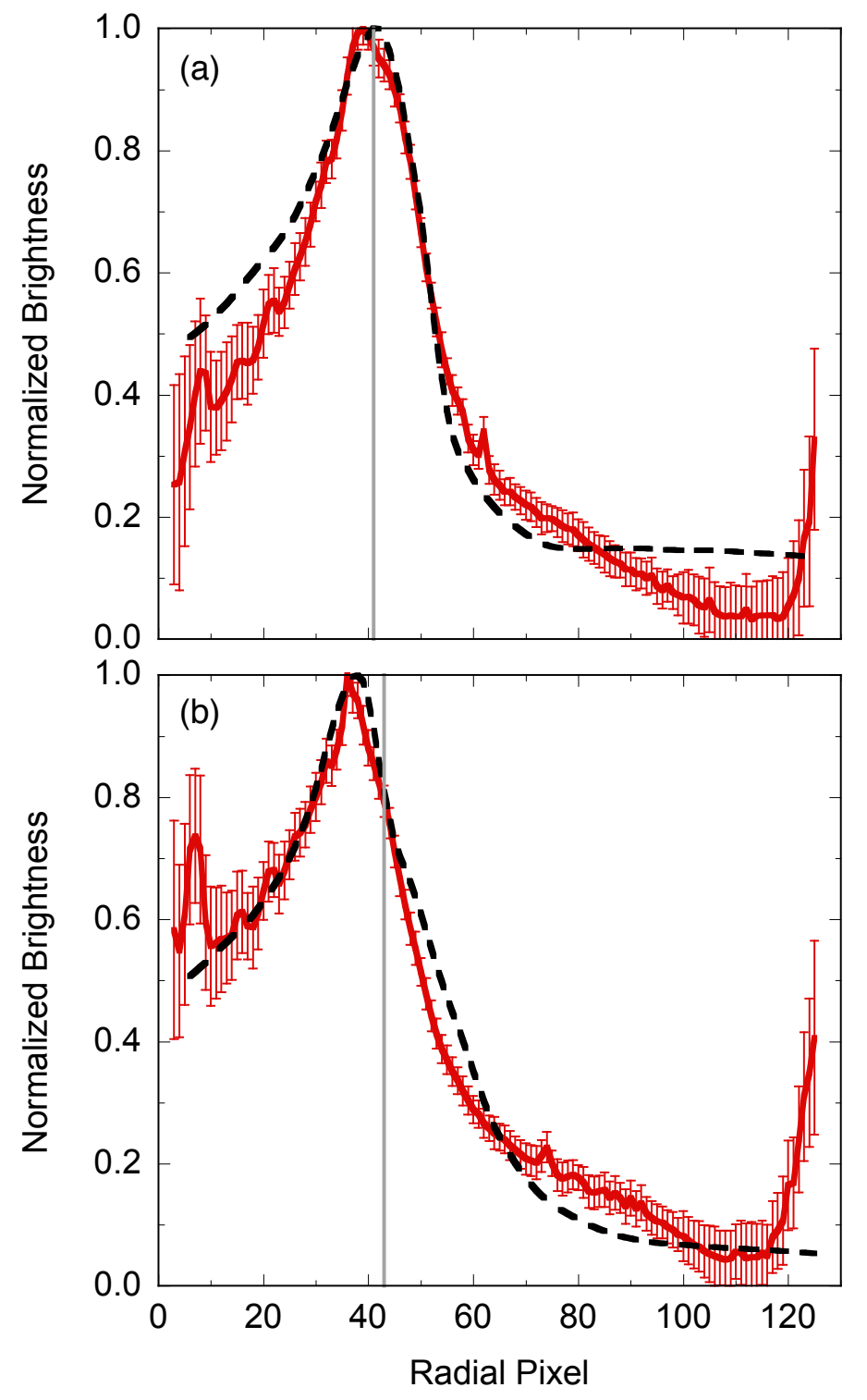

Figure 2: Simulated (dashed) and observed (solid) radial ENDD profiles of line integrated Balmer- $\beta$ brightness for shots (a) 139412 and (b) 142214, all normalized to maximum values of unity. The horizontal axis corresponds to a spatial extent of $\sim 20 \mathrm{~cm}$; the gray lines indicate the pixels closest to the separatrix. The error bars on the experimental profile are associated with the calibration of the ENDD camera. 


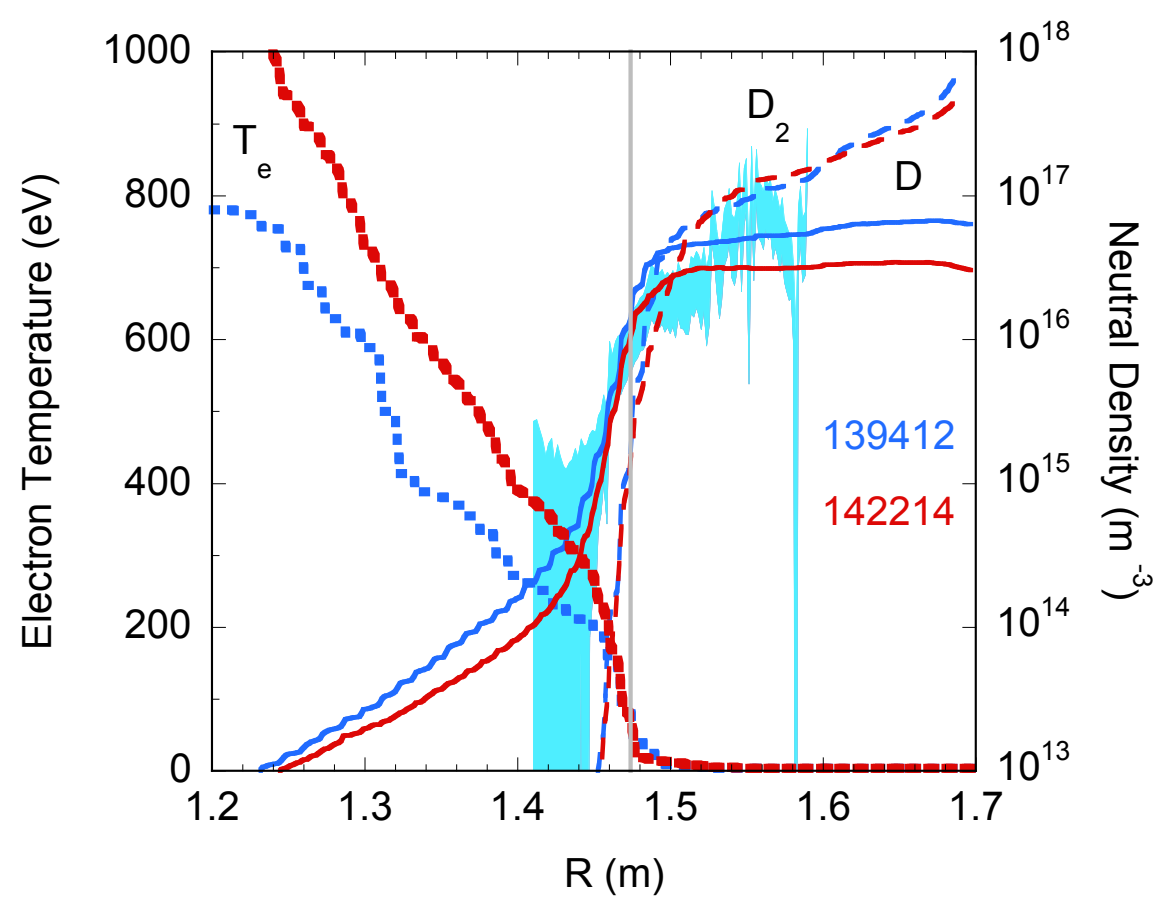

Figure 3: Inferred deuterium atom (solid lines) and molecule (dashed lines) midplane density profiles for NSTX shots 139412 and 142214, both at 0.4 s. The electron temperature profiles (dotted lines) are provided for reference. The gray line indicates the separatrix location (same for both shots). The shaded area represents the atom density for shot 142214 inferred from the inverted ENDD signal via Eq. (2); the vertical width indicates the propagated uncertainty associated with the ENDD camera calibration. 